\title{
Study on interaction between phosphorus and cadmium in sewage sludge during hydrothermal treatment by adding hydroxyapatite
}

Wansheng Shi ${ }^{\mathrm{a}, \mathrm{b}}$, Chuanping Feng ${ }^{\mathrm{a}}$, Wenli Huang ${ }^{\mathrm{b}}$, Zhongfang Lei ${ }^{\mathrm{b} *}$, Zhenya Zhang ${ }^{\mathrm{b}}$

${ }^{a}$ School of Water Resources and Environment, China University of Geosciences, Beijing 100083, China

${ }^{\mathrm{b}}$ Graduate School of Life and Environmental Sciences, University of Tsukuba, Tsukuba 3058572, Japan

*Corresponding author. Tel/fax: +81 29853 6703. E-mail: lei.zhongfang.gu@u.tsukuba.ac.jp (Z. Lei); zhang.zhenya.fu@u.tsukuba.ac.jp (Z.Zhang). 


\begin{abstract}
This study focused on the behavior of phosphorus $(\mathrm{P})$ and its effect on cadmium $(\mathrm{Cd})$ immobilization in sludge during HTT. The results showed after HTT other forms of P in sludge were almost completely converted into apatite P (AP) and the eco-toxicity and leachability of Cd was sharply decreased, signaling the immobilization effect of HTT on Cd in the sludge. Hydroxyapatite (HAP) addition showed a synergetic effect with HTT on Cd risk reduction, implying the immobilization effect of HTT on Cd could be partly attributed to the strong binding capacity of phosphorus with $\mathrm{Cd}$. P content was found to have a strong relationship with the existing forms of Cd during HTT. The present results suggest that HTT with HAP addition could be a promising method for the safe disposal of Cd-contaminated sludge.
\end{abstract}

Keywords: Sewage sludge; Hydrothermal treatment (HTT); Hydroxyapatite (HAP); Cadmium (Cd); Immobilization 


\section{Introduction}

A huge amount of sewage sludge is annually produced worldwide, which urgently demands a safe, efficient, and sustainable treatment and disposal method. The best disposal method should not only take full use of the nutrient sources in the sludge but also minimize its potential environmental risk brought by the pollutants contained. Landfill, incineration and land use are known to be the main methods used to cope with sewage sludge at present (Lederer and Rechberger, 2010). Landfill is very simple to treat sewage sludge but it is a kind of waste of nutrient sources and land, which also brings about some secondary pollution. Incineration is not a cost-effective way and causes some air pollution, especially the produced fly ash which is very difficult to deal with because of containing hazardous and high concentration of heavy metals (HMs) (Dewil et al., 2007). Therefore, from a long run, land use may be the best alternative for sewage sludge disposal (Deng et al., 2009; Babel and del Mundo Dacera, 2006).

In order to minimize the environmental risk of pathogens and some other pollutants (especially HMs) in the sewage sludge before land use, appropriate pre-treatment is crucial (Tao et al., 2012; Zhu et al., 2013). Carbonization is now widely recognized as an environmentally friendly way because the produced biochar could provide some easily available nutrient sources, such as N, P and K, which can be used to improve the productivity of soil (Khan et al., 2013a). Moreover, biochar can also provide a kind of additive for the remediation of contaminated soil with its strong binding capacity for some HMs (Méndez et al., 2012) and trace organic pollutants (such as PAH, herbicide, pesticide, etc.) (Khan et al., 2013b).

To date research works are mainly focused on the carbonization of sewage sludge through dry pyrolysis under high temperature conditions (usually $>300^{\circ} \mathrm{C}$ ) and the sludge drying process 
before pyrolysis requires large amount of energy. Recently, hydrothermal treatment (HTT) has been investigated and used for solid waste treatment and is recognized as a sustainable method (Lu et al., 2012; Weiner et al., 2013; Parshetti et al., 2013). Before HTT process the dehydration and drying process is not required. Furthermore, the dehydration performance of the sludge was greatly improved after HTT process (Shi et al., 2013).

In order to identify the safety of the produced hydrochar before soil application, the risk of HMs should be evaluated and minimized. Previous research indicates that the risk of HMs in the sludge may be changed when the evaluation is made by total concentration and existing forms after HTT process (Hossain et al., 2011). In addition, some metals are found to be mainly accumulated in the produced hydrochar but their existing forms have been transformed from weakly bound forms to more stable state after this process (Shi et al., 2013). Up to now, the fractional transformation is regarded to be the major reason resulting in the immobilization and toxicity reduction of HMs in the sludge. The related mechanism, however, remains unclear.

Furthermore, during HTT the existing forms of other elements in the sludge would also be changed, especially phosphorus (Zhu et al., 2011). Importantly, the existing forms of phosphorus are closely related not only to their bioavailability in soil but also to the environmental risk of some metals bound with phosphorus in the sludge (Chen et al., 2007). Based on its binding capacity with HMs, some trial has been carried out on the effectiveness of phosphorus (by using phosphoric acid and monobasic calcium phosphate) on HMs immobilization in tannery sludge after incineration (Tang et al., 2013). In addition, some other phosphorus forms (mainly phosphate and phosphate-based minerals) are also found to have immobilization effect on HMs and can be used for the reclamation of contaminated soils by HMs (McGowen et al., 2001; Wu et al., 2013; Chen et al., 2006). Therefore, it is speculated that the behavior of phosphorus may 
affect the fractional transformation of HMs during HTT. In order to confirm this speculation, in this study Cd was selected, due to its high toxicity and existed widely in sludge, to investigate the behavior of phosphorus and its interaction with $\mathrm{Cd}$ immobilization during HTT process. The effect of phosphorus on the environmental risk and toxicity of $\mathrm{Cd}$ in sludge during HTT was also disclosed.

\section{Materials and methods}

\subsection{Sewage sludge}

The sewage sludge used in this experiment was dewatered anaerobically digested sewage sludge cake obtained from a wastewater treatment plant in Ibaraki prefecture, Japan. The moisture content of the sludge was adjusted by adding deionized water, and then agitated so as to be homogeneously mixed. An appropriate volume of Cd (II) (standard Cd solution, 1000mg/l, pH 6.61) was added into the sludge mixture to elevate the content of $\mathrm{Cd}$ because of its low $\mathrm{Cd}$ content in the original sludge. Then the mixture was once more agitated to be homogeneously mixed. After that, the mixture was kept in $4^{\circ} \mathrm{C}$, and intermittently agitated once every day in order to make the added $\mathrm{Cd}$ be homogeneously distributed in the sludge. Table 1 lists the main characteristics of the sludge used in this study.

\subsection{Hydrothermal treatment}

HTT experiments were carried out in a stainless steel reactor with a volume of $200 \mathrm{ml}$. The prepared sludge sample (about $100 \mathrm{~g}$ to keep the same volume) was filled into this reactor and heated to $200^{\circ} \mathrm{C}$ (HTT200) or $280^{\circ} \mathrm{C}(\mathrm{HTT} 280)$, and then treated at the designated temperature

for $1 \mathrm{~h}$. After cooling to room temperature, the solid residues (SRs) and process water (PW) were 
separated by vacuum filtration. The obtained SRs were dried at $60^{\circ} \mathrm{C}$ for 2 days, then ground into fine powders and mixed homogeneously, and stored in enclosed plastic bags, which were named SR200 and SR280. The obtained process water were respectively labeled as PW200 and PW280, and then kept in refrigerator at $4^{\circ} \mathrm{C}$.

In the control experiment (without HTT process), the prepared sewage sludge was directly dried at $60^{\circ} \mathrm{C}$ for 2 days. The solid residue collected was labeled as SRC, and no process water was obtained.

\subsection{Fractionation of phosphorus}

Phosphorus in sludge can be fractionated into different categories by different sequential extraction protocol (Ruban et al., 2001; Ruban et al., 1999). The most widely used method is the Standards, Measurements and Testing (SMT) Programme extraction protocol (Medeiros et al., 2005; Ruban et al., 2001). After sequential extraction based on the SMT method, phosphorus in sludge can be fractionated into the following 5 categories: (1) concentrated $\mathrm{HCl}$-extractable $\mathrm{P}$, namely total P (TP), (2) organic P (OP), (3) inorganic P (IP), (4) non-apatite inorganic P (NAIP, the $\mathrm{P}$ fraction associated with oxides and hydroxides of $\mathrm{Al}, \mathrm{Fe}$ and $\mathrm{Mn}$ ), and (5) apatite $\mathrm{P}(\mathrm{AP}$, the $\mathrm{P}$ fraction associated with $\mathrm{Ca}$ ). The phosphorus concentration in the supernatant collected after extraction was determined with spectrophotometric method.

\subsection{Risk assessment of $C d$ in sludge}

\subsubsection{Total concentration}


In order to minimize the environmental risk and toxicity, HMs in sludge should meet stringent standards which are mainly based on their total concentrations. Therefore, total concentration was used in this study as one of the major indices for the assessment of HMs risk in the sludge.

\subsubsection{Fractionation of $C d$ and risk assessment code (RAC)}

On the other hand, it's widely recognized that the risk or eco-toxicity of HMs in sludge is determined not only by the total concentration but also by their existing forms (Xian, 1989), most probably attributable to the fact that HMs in different existing forms possess different binding strength and stability in sludge residue, resulting in the difference in their possible mobility, toxicity and bioavailability (Vela et al., 1993).

$\mathrm{Cd}$ in sludge can be fractionated into 5 fractions by using the sequential extraction procedure proposed by Tessier et al. (1979) with $1.0 \mathrm{~g}$ sample. The five fractions are exchangeable Cd (F1), carbonate bound $\mathrm{Cd}(\mathrm{F} 2)$, iron and manganese oxides bound $\mathrm{Cd}(\mathrm{F} 3)$, Cd bound to organic and sulfide (F4) and Cd in the residue state (F5). After extraction, the collected samples for Cd in F1F4 were acidified, filtered and then digested before determination. The samples for the determination of $\mathrm{Cd}$ in F5 and total Cd concentration were extracted by acid digestion with a mixture of $\mathrm{HCl}-\mathrm{HNO}_{3}-\mathrm{HF}(3: 1: 1, \mathrm{v} / \mathrm{v})$ in PTFE beakers on a heating plate.

$\mathrm{Cd}$ in different factions possesses different mobility, eco-toxicity and bioavailability. Based on the binding strength and availability, the fractions of $\mathrm{Cd}$ in sludge can be grouped into 3 categories as $(\mathrm{F} 1+\mathrm{F} 2),(\mathrm{F} 3+\mathrm{F} 4)$ and $\mathrm{F} 5$, respectively, reflecting the weakly bound or easily releasable $\mathrm{Cd}$, relatively stable $\mathrm{Cd}$ and the $\mathrm{Cd}$ remained in the sludge matrix or resultant hydrochar. These 3 parts of $\mathrm{Cd}$ are reported to exhibit different eco-toxicity and biovailability in 
the environment as direct toxicity $(\mathrm{F} 1+\mathrm{F} 2)$, potential toxicity $(\mathrm{F} 3+\mathrm{F} 4)$ and no toxicity $(\mathrm{F} 5)$, respectively (Li et al., 2012).

Furthermore, the risk of $\mathrm{Cd}$ in sludge can also be evaluated by the weakly bound fractions $(\mathrm{F} 1+\mathrm{F} 2)$, because $\mathrm{Cd}$ in these fractions are easily affected by ionic strength and vulnerable to $\mathrm{pH}$ changes in soil environment resulting in a very high mobility (Lasheen and Ammar, 2009).

Based on this consideration, the specific quantity evaluation of $\mathrm{Cd}$ in sludge can be realized by using a risk assessment code (RAC) according to the proportion of these two fractions (F1+F2) to the total amount of Cd extracted from the sludge, namely the risk index (RI) (Singh et al., 2005). Then Cd in sludge can be classified by using RAC as no risk, low risk, medium risk, high risk, and very high risk when the RI value ranges $<1,1-10,11-30,31-50$, and $>50$, respectively.

\subsubsection{Toxicity characteristic leaching procedure (TCLP)}

Leachability is another important parameter for assessing the mobility and bioavailability of Cd in sludge. In this study, the U.S. EPA TCLP leaching procedure (USEPA, 1997) was applied. The leaching tests of $\mathrm{Cd}$ were carried out by extraction with acetic acid solution at $\mathrm{pH} 2.88$, liquid/solid ratio of 20:1, and extraction for $18 \mathrm{~h}$. After extraction, the supernatant collected by centrifugation was filtered, acidified and digested for Cd determination.

\subsection{Effect of phosphorus on behavior of Cd during HTT}

In order to investigate the effect of phosphorus in sludge on the behavior of Cd during HTT, hydroxyapatite (HAP) was added into the HTT system based on our preliminary results that phosphorus was mainly existed in the tested sludge in the form of AP (Table 2). The differences of $\mathrm{Cd}$ in redistribution, fractionation and leachability before and after HAP addition were examined and used to indicate the effect of phosphorus on Cd behavior during HTT. HAP was 
added into the HTT reactor with the dosage of $0 \%, 10 \%$ and $40 \%$ (P basis) according to the total phosphorus content in the sludge (i.e. HAP-P/TP sludge $=0 \%, 10 \%$ and $40 \%$, w/w, respectively). After HTT at $200^{\circ} \mathrm{C}$ or $280^{\circ} \mathrm{C}, \mathrm{Cd}$ in the obtained process water and solid residue were analyzed.

\subsection{Analysis}

The concentration of each metal was determined by using ICP-MS (ELAN DRC-e, PerkinElmer). Phosphorus was determined by ammonium molybdate spectrophotometric method. Each test was conducted in triplicate and the results were expressed as mean values $\pm \mathrm{SD}$.

\section{Results and discussions}

\subsection{Redistribution of phosphorus and major metals in sludge after HTT process}

HTT usually has both extraction and stabilization effects for some inorganics and therefore leads to redistribution of these elements (Escala et al., 2012; Jin et al., 2012).

Fig. 1 shows the redistribution of phosphorus and the major metals closely related to the existing forms of phosphorus in PW and SR after HTT. A very small part of phosphorus, Ca, Al, Fe and Mn were detected to release into PW $(<0.6 \%)$ while the majority of them were accumulated in the collected SRs, and the extent of this kind of accumulation increased with the increase of treatment temperature. Ca was existed at a very high level (14.84 \%) in the sludge, followed by $\mathrm{Fe}(5.34 \%), \mathrm{Al}(0.73 \%)$ and $\mathrm{Mn}(0.04 \%)$. These four metals may influence the behavior of phosphorus in the sludge during HTT process.

Table 2 shows the changes in fractionation of phosphorus in sludge before and after HTT. The phosphorus was mainly existed in the form of inorganic $\mathrm{P}$ (IP) in the tested sludge and its organic $\mathrm{P}(\mathrm{OP})$ was very low. In addition, apatite $\mathrm{P}$ (AP) was the main form of inorganic $\mathrm{P}$ (IP), 
possibly ascribing to a higher content of $\mathrm{Ca}$ and relatively lower contents of $\mathrm{Fe}, \mathrm{Al}$ and $\mathrm{Mn}$ (Fig. 1).

After HTT, significant increase was found in the content of inorganic P with the treatment temperature increased from $200^{\circ} \mathrm{C}$ to $280^{\circ} \mathrm{C}$, while sharp decrease occurred correspondingly to the organic $\mathrm{P}$ fraction in the sludge residue, signaling the organic $\mathrm{P}$ could almost be converted into inorganic P. Furthermore, non-apatite inorganic P was detected to decrease after HTT process in spite of the increase in inorganic $\mathrm{P}$ fraction, indicating phosphorus was mainly transformed from NAIP to AP. This conversion of phosphorus into AP could be resulted not only from the high content of $\mathrm{Ca}$ in the tested sludge to form apatite, but also from the large increase in $\mathrm{pH}$ value of the sludge mixture in the reactor from 6.4 (original tested sludge) to 8.7 (PW200) and 9.4 (PW280) after HTT process. The resultant alkaline condition favored the dissolution of NAIP (Eq. 1) and the released $\mathrm{PO}_{4}{ }^{3-}$ could combine with Ca to form the relatively stable Ca bound P (Eq. 2) (Petzet et al., 2012).

$$
\begin{aligned}
& \mathrm{AlPO}_{4}+4 \mathrm{OH}^{-} \leftrightarrow\left[\mathrm{Al}(\mathrm{OH})_{4}\right]^{-}+\mathrm{PO}_{4}^{3-} \\
& 2 \mathrm{PO}_{4}^{3-}+3 \mathrm{Ca}^{2+} \rightarrow \mathrm{Ca}_{3}\left(\mathrm{PO}_{4}\right)_{2}
\end{aligned}
$$

\subsection{Effect of phosphorus on redistribution of $C d$}

After HTT process, the treated sludge was separated into PW and SR, in which the content of Cd was analyzed with the results shown in Fig. 2. A very small part of $\mathrm{Cd}$ was observed to release into PW after HTT process $(<0.2 \%)$ with the majority left in the SRs, meaning that HTT brought about the redistribution of $\mathrm{Cd}$ in these two phases. The accumulated amount of $\mathrm{Cd}$ indicated that the potential environmental risk of $\mathrm{Cd}$ in sludge was increased after HTT when assessing the risk of $\mathrm{Cd}$ by the total concentration in SRs. 
Fig. 2 also shows the effect of HAP addition on the redistribution of Cd in PW and SRs. From Fig. $2 b$ it can be seen that when HAP was added into the tested sludge, the concentration of Cd decreased slightly in the sludge before HTT process, most probably due to a dilution effect because of HAP addition. During HTT process, HAP addition caused an obvious reduction in the content of Cd released into PW (Fig. 2(a)) while a slight increase in the content of Cd in SRs (Fig. 2(b)), probably contributed by the strong binding capacity of HAP with Cd.

The above results indicated that the potential risk of $\mathrm{Cd}$ was increased to some extent after HTT process with or without HAP addition when evaluated by total Cd concentration in the treated sludge (sludge residue).

\subsection{Effect of phosphorus on fractionation of $C d$}

After HTT process, the fractionation of Cd in sludge was determined by sequential extraction, and the result is shown in Fig. 3. The content of $\mathrm{Cd}$ in different fractions can be used to reflect its eco-toxicity due to these existing forms are closely related to its mobility and bioavailability in sludge during soil application. The result indicated that more than half of $\mathrm{Cd}$ was bound to iron and manganese oxides $(\mathrm{F} 3,53.76 \%)$, followed by organic and sulfide bound fraction $(\mathrm{F} 4$, 24.24\%), indicating that $\mathrm{Cd}$ was mainly existed in the potential toxicity forms (F3+F4) with very small part in exchangeable (F1) and residue state (F5).

Hydrothermal treatment can induce the decomposition of organics in sludge and redistribution of $\mathrm{Cd}$, and then alter the fractionation of $\mathrm{Cd}$ in the treated sludge. This fractional transformation of $\mathrm{Cd}$ can be discerned from Fig. 3. After HTT, Cd in the first 2 factions was sharply decreased while $\mathrm{Cd}$ in the last 2 fractions (F4 and F5) was increased with the increase of treatment temperature. $\mathrm{Cd}$ in $\mathrm{F} 3$ was first slightly increased $\left(200^{\circ} \mathrm{C}\right)$ and then largely decreased $\left(280{ }^{\circ} \mathrm{C}\right)$, 
likely attributable to the thermostability of $\mathrm{Cd}$ binding with $\mathrm{Fe}$ and $\mathrm{Mn}$ oxides. The fractional transformation of $\mathrm{Cd}$ from weakly bound forms (F1 and F2) to a relatively stable form (F4) and stable state (F5) implies the immobilization and risk reduction effect of HTT on Cd in the sludge.

Furthermore, HAP addition can enhance this immobilization effect of $\mathrm{Cd}$ thus achieving its risk reduction in sludge after HTT process. The risk reduction of $\mathrm{Cd}$ in the sludge can be clearly observed after directly drying the sludge (SRC) and HTT process (SR200and SR280) with HAP addition (Fig. 3). The content of $\mathrm{Cd}$ in the first 3 fractions decreased while $\mathrm{Cd}$ in $\mathrm{F} 4$ increased with HAP addition almost in a dose-dependent manner. Cd in F5 decreased slightly in SRC and SR280 while significantly increased in SR200 with HAP addition. Anyhow, HAP addition showed a positive effect on immobilization and risk reduction of $\mathrm{Cd}$ by transforming $\mathrm{Cd}$ into more stable state which may have strong binding affinity of phosphate.

For the quantitative risk evaluation of $\mathrm{Cd}, \mathrm{RAC}$ method based on $\mathrm{Cd}$ in fractions $(\mathrm{F} 1+\mathrm{F} 2)$ can be used to reflect its direct eco-toxicity, mobility and bioavailability. The results in Fig. 3 show that $\mathrm{Cd}$ in the tested sludge exhibited medium risk to the environment. After $\mathrm{HTT}$ at $200^{\circ} \mathrm{C}$ and $280^{\circ} \mathrm{C}, \mathrm{Cd}$ in $(\mathrm{F} 1+\mathrm{F} 2)$ decreased by $95 \%$ and $98 \%$, respectively, thus the risk of $\mathrm{Cd}$ in SRs was sharply decreased from medium risk (in the original tested sludge) to low risk (in SR200) and no risk (in SR280). When HAP was added, $\mathrm{Cd}$ in fraction $(\mathrm{F} 1+\mathrm{F} 2)$ decreased with the increase in HAP dosage both in the drying and HTT processes, showing HAP addition had a synergetic effect with HTT process on reduction in the direct toxicity of Cd. Fig. 3 also shows that this synergetic effect was more significantly when HTT was conducted under $200^{\circ} \mathrm{C}$.

Table 3 lists the correlation coefficient ( $\mathrm{r}$ ) between factions of $\mathrm{Cd}$ and phosphorus content in the sludge after HTT process. Cd in F1 and F3 were found to have a strongly negative relationship with P content in the sludge, while a strongly positive relationship existed between 
F4 and P content in the sludge. Specifically, under HTT of $200^{\circ} \mathrm{C}$ condition all Cd fractions were found to have strong relationship with $\mathrm{P}$ concentration, indicating $200^{\circ} \mathrm{C}$ might be the crucial temperature when taking the stability of formed Cd-sludge matrix, especially the binding stability of $\mathrm{Cd}$ with $\mathrm{Fe}$ and Mn oxides into consideration.

In contrast, no clear relationship could be concluded with respect to phosphorus content and the fractions of $\mathrm{Cd}$ in F2 and F5 in the sludge, which needs further investigation.

The result clearly indicates that phosphorus plays a very important role on the fractional transformation of $\mathrm{Cd}$ in sludge during HTT process. The strong relationship between $\mathrm{Cd}$ fractions (especially F1, F3, and F4) and phosphorus content in sludge implies that the effect of HTT on Cd immobilization is partly contributed by the strong binding capacity of phosphorus in the sludge matrix.

\subsection{Effect of phosphorus on leachability of $C d$}

The risk of $\mathrm{Cd}$ in sludge can also be reflected directly by its leachability which is closely related to its mobility. The leaching toxicity of $\mathrm{Cd}$ in sludge was determined with the standard TCLP test. The leaching concentration of Cd from the sludge residue is shown in Fig. 4.

Lower than $1.0 \mathrm{mg} \mathrm{kg}^{-1}$ of $\mathrm{Cd}$ was detected to be released into the solution, and the leached content of Cd was sharply decreased from $2.0 \%$ (in SRC) to $0.13 \%$ (in SR200) and $0.08 \%$ (in SR280) after HTT process, respectively. Therefore, Cd in sludge was definitely immobilized after HTT process from the leachability test results, and the toxicity of $\mathrm{Cd}$ was decreased with the increase of treatment temperature, showing the positive effect of HTT on reducing the leaching toxicity and leachability of Cd in sludge. 
Besides, HAP addition further reduced the leaching content of Cd from the SRs collected from both drying and HTT processes. More reduction in leaching toxicity was observed after HAP addition, also signaling the synergetic effect of phosphorus and HTT on reducing the toxicity of Cd in sludge.

\subsection{Implication of this study to practice}

In this study, the behavior of Cd during $\mathrm{HTT}$ under $200^{\circ} \mathrm{C}$ and $280^{\circ} \mathrm{C}$ was investigated along with its interaction with phosphorus in sludge by HAP addition. The experimental results showed that although the total Cd concentration increased to some extent in the solid residues after HTT process, the risk of $\mathrm{Cd}$ was found to decrease largely when evaluated by its existing forms and leachability, indicating the feasibility of using HTT for the disposal of sewage sludge in practice. Furthermore, HAP addition can add this $\mathrm{Cd}$ immobilization effect by further reducing the risk and toxicity of Cd in sludge. Restated, the observed synergetic effect implies that the phosphorus existed in the sludge to a certain degree should have some contribution to $\mathrm{Cd}$ immobilization in the sludge during HTT. Furthermore, HAP addition may provide a new applicable method for enhancing the HMs immobilization in sludge by HTT process, providing a safe and sustainable treatment method for HMs-contaminated sludge before land use.

\section{Conclusion}

This study presented a promising method for the enhancement of Cd immobilization in sludge through HTT process. During HTT, phosphorus was clearly found to be converted into apatite P from other fractions, and Cd was immobilized in the treated sludge with its toxicity and risk being greatly reduced. The present results also indicate that HAP addition has a synergetic 
immobilization effect on the redistribution, fractionation and leachability of $\mathrm{Cd}$, implying that the immobilization of Cd during HTT process can be partly ascribed to the phosphorus in the sludge due to its strong binding affinity with $\mathrm{Cd}$.

\section{References}

1. Babel, S., del Mundo Dacera, D., 2006. Heavy metal removal from contaminated sludge for land application: A review. Waste Manage. 26, 988-1004.

2. Chen, S. B., Xu, M. G., Ma, Y. B., Yang, J. C., 2007. Evaluation of different phosphate amendments on availability of metals in contaminated soil. Ecotoxicol. Environ. Saf. 67, 278-285.

3. Chen, S. B., Zhu, Y. G., Ma, Y. B., 2006. The effect of grain size of rock phosphate amendment on metal immobilization in contaminated soils. J. Hazard. Mater.134, 74-79.

4. Deng, J., Feng, X., Qiu, X., 2009. Extraction of heavy metal from sewage sludge using ultrasound-assisted nitric acid. Chem. Eng. J. 152, 177-182.

5. Dewil, R., Baeyens, J., Appels, L., 2007. Enhancing the use of waste activated sludge as biofuel through selectively reducing its heavy metal content. J. Hazard. Mater.144, 703-707.

6. Escala, M., Zumbühl, T., Koller, C., Junge, R., Krebs, R., 2012. Hydrothermal Carbonization as an Energy-Efficient Alternative to Established Drying Technologies for Sewage Sludge: A Feasibility Study on a Laboratory Scale. Energy Fuels 27, 454-460.

7. Hossain, M. K., Strezov, V., Chan, K. Y., Ziolkowski, A., Nelson, P. F., 2011. Influence of pyrolysis temperature on production and nutrient properties of wastewater sludge biochar. J. Environ. Manage. 92, 223-228. 
8. Jin, Y. Q., Ma, X. J., Jiang, X. G., Liu, H. M., Li, X. D., Yan, J. H., Cen, K. F., 2012. Effects of Hydrothermal Treatment on the Major Heavy Metals in Fly Ash from Municipal Solid Waste Incineration. Energ. Fuel. 27, 394-400.

9. Khan, S., Chao, C., Waqas, M., Arp, H. P. H., Zhu, Y. G., 2013a. Sewage sludge biochar influence upon rice (Oryza sativa L) yield, metal bioaccumulation and greenhouse gas emissions from acidic paddy soil. Environ. Sci. Technol. 47, 8624-8632.

10. Khan, S., Wang, N., Reid, B. J., Freddo, A., Cai, C., 2013b. Reduced bioaccumulation of PAHs by Lactuca satuva L. grown in contaminated soil amended with sewage sludge and sewage sludge derived biochar. Environ. Pollut. 175, 64-68.

11. Lasheen, M. R., Ammar, N. S., 2009. Assessment of metals speciation in sewage sludge and stabilized sludge from different Wastewater Treatment Plants, Greater Cairo, Egypt. J. Hazard. Mater. 164, 740-749.

12. Lederer, J., Rechberger, H., 2010. Comparative goal-oriented assessment of conventional and alternative sewage sludge treatment options. Waste Manage. 30, 1043-1056.

13. Li, L., Xu, Z. R., Zhang, C. L., Bao, J. P., Dai, X. X., 2012. Quantitative evaluation of heavy metals in solid residues from sub- and super-critical water gasification of sewage sludge. Bioresour. Technol. 121, 169-175.

14. Lu, X., Jordan, B., Berge, N. D., 2012. Thermal conversion of municipal solid waste via hydrothermal carbonization: Comparison of carbonization products to products from current waste management techniques. Waste Manage. 32, 1353-1365.

15. Méndez, A., Gómez, A., Paz-Ferreiro, J., Gascó, G., 2012. Effects of sewage sludge biochar on plant metal availability after application to a Mediterranean soil. Chemosphere 89, 13541359. 
16. McGowen, S. L., Basta, N. T., Brown, G. O., 2001. Use of diammonium phosphate to reduce heavy metal solubility and transport in smelter-contaminated soil. J. Environ. Qual. 30, 493-500.

17. Medeiros, J. J. G., Cid, B. P., Gomez, E. F., 2005. Analytical phosphorus fractionation in sewage sludge and sediment samples. Anal. Bioanal. Chem. 381, 873-878.

18. Parshetti, G. K., Liu, Z., Jain, A., Srinivasan, M. P., Balasubramanian, R., 2013. Hydrothermal carbonization of sewage sludge for energy production with coal. Fuel 111, 201-210.

19. Petzet, S., Peplinski, B., Cornel, P., 2012. On wet chemical phosphorus recovery from sewage sludge ash by acidic or alkaline leaching and an optimized combination of both. Water Res. 46, 3769-3780.

20. Ruban, V., Lopez-Sanchez, J. F., Pardo, P., Rauret, G., Muntau, H., Quevauviller, P., 1999. Selection and evaluation of sequential extraction procedures for the determination of phosphorus forms in lake sediment. J. Environ. Monit. 1, 51-56.

21. Ruban, V., Lopez-Sanchez, J. F., Pardo, P., Rauret, G., Muntau, H., Quevauviller, P., 2001. Harmonized protocol and certified reference material for the determination of extractable contents of phosphorus in freshwater sediments - A synthesis of recent works. Fresenius J. Anal. Chem. 370, 224-228.

22. Shi, W., Liu, C., Ding, D., Lei, Z., Yang, Y., Feng, C., Zhang, Z., 2013. Immobilization of heavy metals in sewage sludge by using subcritical water technology. Bioresour. Technol. $137,18-24$. 
23. Singh, K. P., Mohan, D., Singh, V. K., Malik, A., 2005. Studies on distribution and fractionation of heavy metals in Gomti river sediments - a tributary of the Ganges, India. J. Hydrol. 312,14-27.

24. Tang, P., Zhou, Y. C., Xie, Z. M., 2013. Immobilization of heavy metals in sludge using phosphoric acid and monobasic calcium phosphate. J. Zhejiang Univ. Sci. A 14, 177-186.

25. Tao, J., Wu, S., Sun, L., Tan, X., Yu, S., Zhang, Z., 2012. Composition of waste sludge from municipal wastewater treatment plant. Procedia Environ. Sci. 12, 964-971.

26. Tessier, A., Campbell, P. G. C., Bisson, M., 1979. Sequential Extraction Procedure for The Speciation of Particulate Trace-Metals. Anal. Chem. 51, 844-851.

27. USEPA, 1997. Test methods for evaluating solid waste - physical chemical methods. SW846. Washington, DC: U.S. Environmental Protection Agency.

28. Vela, L. D., Jervis, R. E., Krishnan, S. S., 1993. The leachability of elements in solid- wastes. J. Radioanal. Nucl. Chem. Art. 169, 39-45.

29. Weiner, B., Baskyr, I., Poerschmann, J. Kopinke, F. D., 2013. Potential of the hydrothermal carbonization process for the degradation of organic pollutants. Chemosphere 92, 674-680.

30. Wu, W. H., Xie, Z. M., Xu, J. M., Wang, F., Shi, J. C., Zhou, R. B., Jin, Z. F., 2013. Immobilization of trace metals by phosphates in contaminated soil near lead/zinc mine tailings evaluated by sequential extraction and TCLP. J. Soil Sediment 13, 1386-1395.

31. Xian, X., 1989. Effect of chemical forms of cadmium, zinc, and lead in polluted soils on their uptake by cabbage plants. Plant Soil 113, 257-264.

32. Zhu, W., Xu, Z. R., Li, L., He, C., 2011. The behavior of phosphorus in sub- and supercritical water gasification of sewage sludge. Chem. Eng. J. 171, 190-196. 
33. Zhu, Y., Zeng, G., Zhang, P., Zhang, C., Ren, M., Zhang, J., Chen, M., 2013. Feasibility of bioleaching combined with Fenton-like reaction to remove heavy metals from sewage sludge. Bioresour. Technol. 142, 530-534. 
Table 1 Physicochemical characteristics of the sludge used in the experiment

\begin{tabular}{|c|c|}
\hline Parameters & Value(Mean $\left.\pm \mathrm{SD}^{\mathrm{a}}\right)$ \\
\hline Moisture content (\%) & $84.98 \pm 0.47$ \\
\hline Organic matter $(\%)$ & $46.25 \pm 0.31$ \\
\hline $\operatorname{Ash}(\%)$ & $53.75 \pm 0.31$ \\
\hline $\mathrm{pH}$ & $6.43 \pm 0.21$ \\
\hline $\mathrm{TN}^{\mathrm{b}}$ ( $\mathrm{g} \mathrm{kg}^{-1}$ dry weight) & $21.06 \pm 0.03$ \\
\hline $\mathrm{TP}^{\mathrm{c}}$ (g kg ${ }^{-1}$ dry weight) & $26.18 \pm 0.35$ \\
\hline $\mathrm{Cd}$ (mg kg ${ }^{-1}$ dry weight) & $48.31 \pm 3.25$ \\
\hline \multicolumn{2}{|c|}{ Major metals in the sludge $\left(\mathrm{g} \mathrm{kg}^{-1}\right.$ dry weight $)$} \\
\hline $\mathrm{Na}$ & $2.85 \pm 0.72$ \\
\hline $\mathrm{K}$ & $2.99 \pm 0.50$ \\
\hline $\mathrm{Mg}$ & $10.22 \pm 1.45$ \\
\hline $\mathrm{Al}$ & $7.33 \pm 2.03$ \\
\hline $\mathrm{Ca}$ & $148.42 \pm 19.13$ \\
\hline $\mathrm{Mn}$ & $0.43 \pm 0.03$ \\
\hline $\mathrm{Fe}$ & $53.35 \pm 0.83$ \\
\hline
\end{tabular}

${ }^{\mathrm{a}} \mathrm{SD}$, standard deviation; ${ }^{\mathrm{b}} \mathrm{TN}$, total nitrogen; ${ }^{\mathrm{c}} \mathrm{TP}$, total phosphorus. 
Table 2 Changes in the existing forms of phosphorus in the solid residues (unit: $\mathrm{mg} \mathrm{g}^{-1}$ )

\begin{tabular}{cccccc}
\hline Sludge residue & NAIP & AP & IP & OP & TP \\
\hline SRC $^{\text {a }}$ & $0.28 \pm 0.01$ & $23.24 \pm 0.26$ & $24.13 \pm 0.28$ & $2.10 \pm 0.02$ & $26.18 \pm 0.35$ \\
SR200 $^{\mathrm{b}}$ & $0.15 \pm 0.02$ & $32.25 \pm 0.11$ & $32.33 \pm 0.22$ & $0.003 \pm 0.00$ & $32.37 \pm 0.34$ \\
SR280 $^{\mathrm{b}}$ & $0.06 \pm 0.02$ & $38.16 \pm 0.18$ & $38.33 \pm 0.54$ & $0.001 \pm 0.00$ & $38.43 \pm 0.26$ \\
\hline
\end{tabular}

${ }^{\mathrm{a}}$ SRC, solid residue as control (sludge before HTT), ${ }^{\mathrm{b}}$ SR200 and SR280 represent solid residues obtained after $\mathrm{HTT}$ at $200^{\circ} \mathrm{C}$ and $280^{\circ} \mathrm{C}$, respectively. 
Table 3 Correlation coefficients (r) between fractions of $\mathrm{Cd}$ and phosphorus content in the sludge

\begin{tabular}{lcllllll}
\hline \multirow{2}{*}{ Samples } & \multirow{2}{*}{ Parameter correlated } & \multicolumn{7}{c}{ Fractions of Cd } \\
\cline { 3 - 8 } & & F1 & F2 & F1+F2 & F3 & F4 & F5 \\
\hline SRC $^{\text {a }}$ & & -0.981 & -0.251 & -0.498 & -0.883 & 0.945 & -0.998 \\
SR200 $^{\mathrm{b}}$ & Phosphorus content & -0.871 & -1.000 & -0.990 & -0.973 & 0.974 & 0.966 \\
SR280 $^{\mathrm{b}}$ & & -0.886 & 0.660 & -0.783 & -0.835 & 0.903 & -0.175 \\
\hline
\end{tabular}

\footnotetext{
${ }^{\mathrm{a}} \mathrm{SRC}$, solid residue control (before HTT), ${ }^{\mathrm{b}}$ SR200 and SR280 represent solid residues obtained after $\mathrm{HTT}$ at $200^{\circ} \mathrm{C}$ and $280^{\circ} \mathrm{C}$, respectively.
} 


\section{Figure captions}

Fig.1. Distribution of total phosphorus and major metals of sludge in process water (PW) (a) and solid residue (SR) (b) before and after HTT process. 200 and 280 indicate the treatment temperature is $200^{\circ} \mathrm{C}$ and $280^{\circ} \mathrm{C}$, respectively; SRC, the solid residue control (i.e., the sludge sample before HTT).

Fig. 2. Redistribution of $\mathrm{Cd}$ in process water (PW) (a) and solid residue (SR) (b) after HTT process with or without HAP addition. 200 and 280 indicate the treatment temperature is $200^{\circ} \mathrm{C}$ and $280^{\circ} \mathrm{C}$, respectively; SRC, the solid residue control (the sludge sample before HTT); HAP$0 \%,-10 \%$ and $-40 \%$ denote the hydroxyapatite addition dosage is $0 \%, 10 \%$ and $40 \%$ ( $\mathrm{P}$ basis, HAP-P/TP $\left.{ }_{\text {sludge, }} \mathrm{w} / \mathrm{w}\right)$, respectively.

Fig. 3. Changes of Cd fractionation in sludge after HTT with or without HAP addition. 200 and 280 indicate the treatment temperature is $200^{\circ} \mathrm{C}$ and $280^{\circ} \mathrm{C}$, respectively; SRC, solid residue control (sludge before HTT); HAP- $0 \%,-10 \%$ and $-40 \%$ denote the hydroxyapatite addition dosage is $0 \%, 10 \%$ and $40 \%$ ( $\mathrm{P}$ basis, $\mathrm{HAP}-\mathrm{P} / \mathrm{TP}_{\text {sludge, }} \mathrm{w} / \mathrm{w}$ ), respectively.

Fig. 4. Leaching concentration of $\mathrm{Cd}$ from sludge residues. 200 and 280 indicate the treatment temperature is $200^{\circ} \mathrm{C}$ and $280^{\circ} \mathrm{C}$, respectively; SRC, the solid residue control (the sludge sample before HTT); HAP- $0 \%,-10 \%$ and $-40 \%$ denote the hydroxyapatite addition dosage is $0 \%, 10 \%$

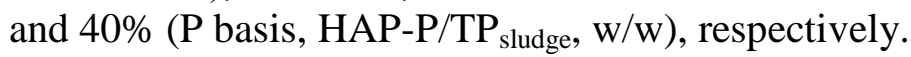




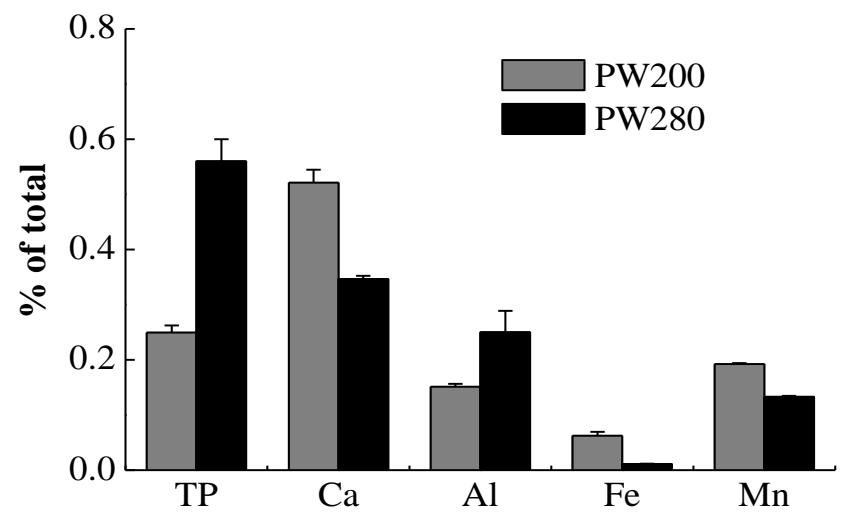

(a)

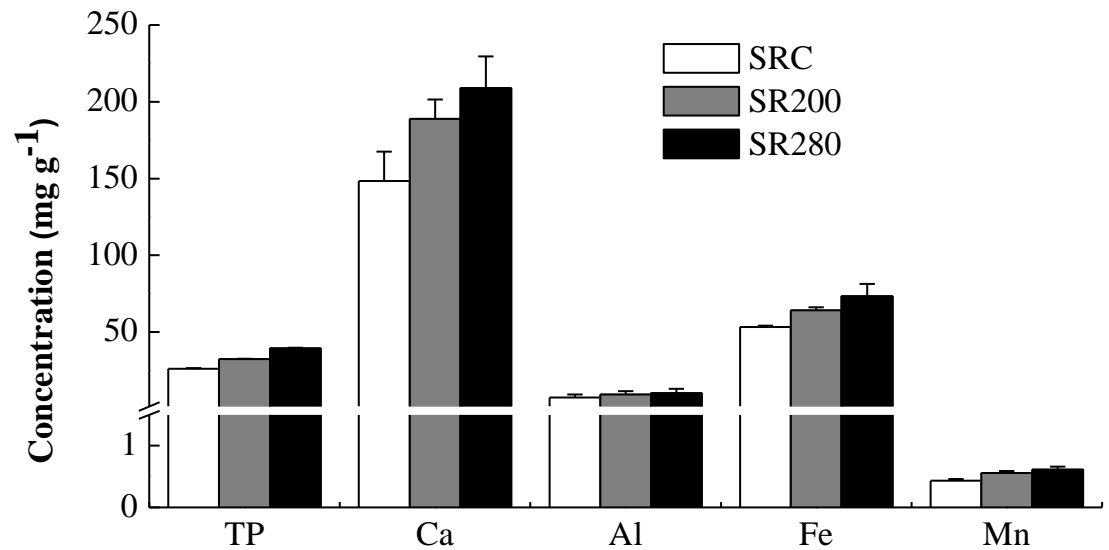

(b)

Fig.1. Shi et al. 


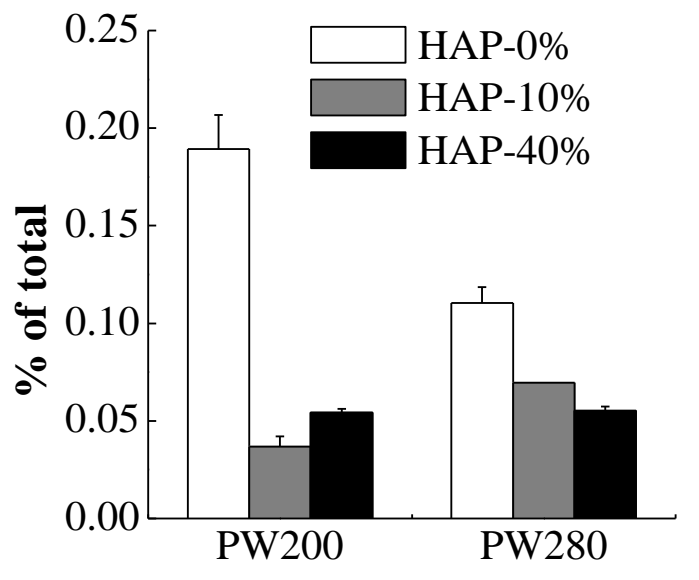

(a)

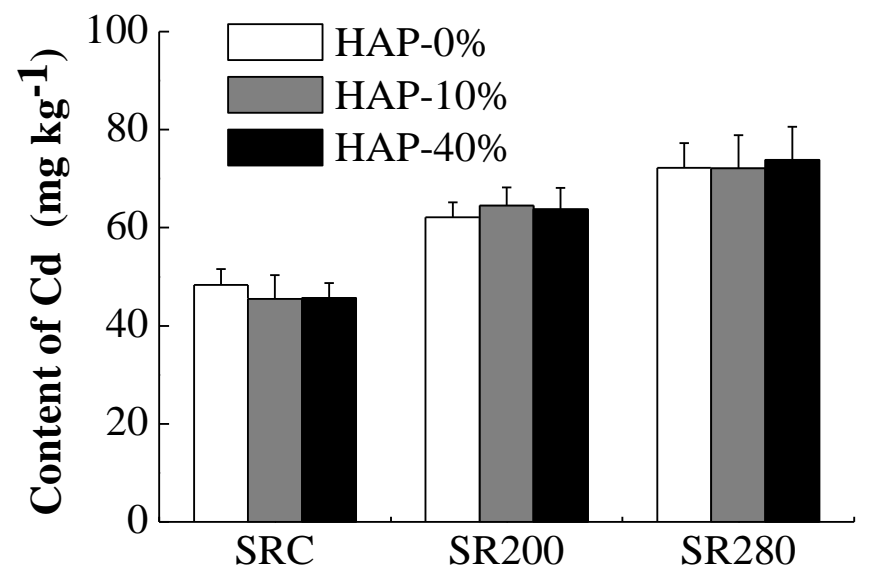

(b)

Fig. 2. Shi et al. 

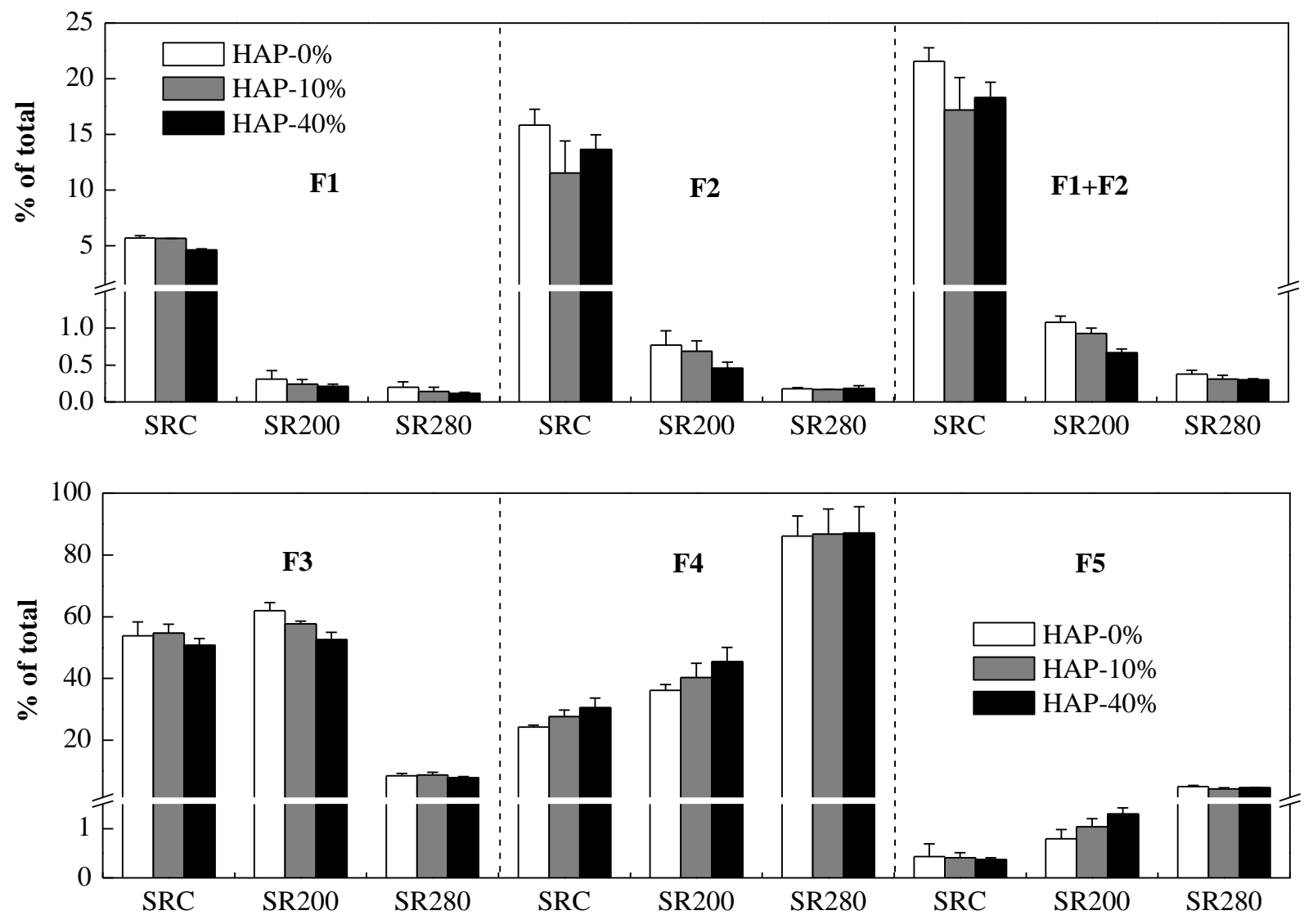

Fig. 3. Shi et al. 


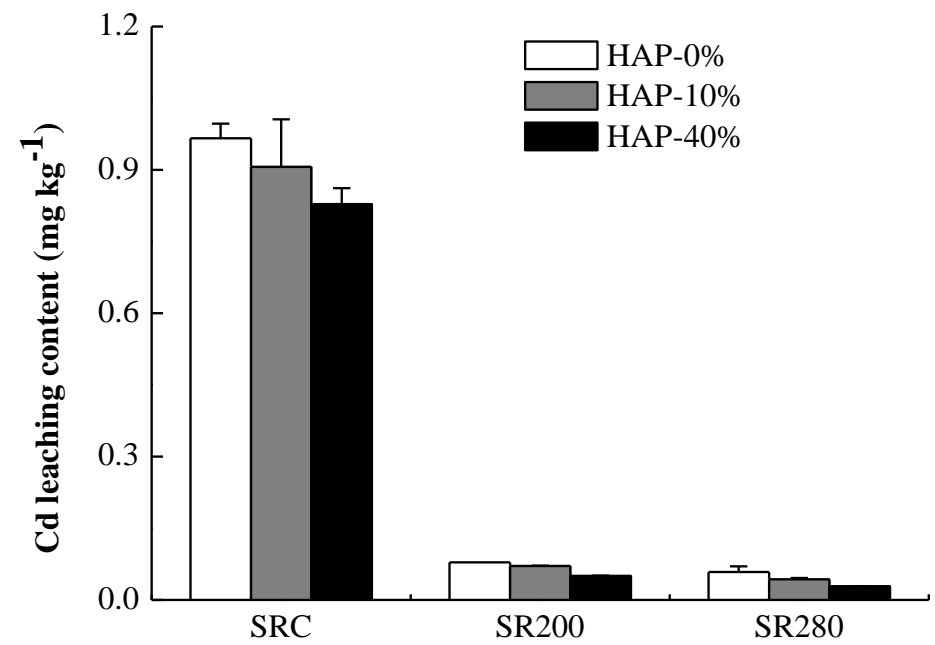

Fig. 4. Shi et al. 